\title{
Functional limitations and associated psychological factors in military personnel with chronic nonspecific neck pain with higher levels of kinesiophobia
}

\author{
Ibai López-de-Uralde-Villanuevaa, a,c,d,*, Ricardo Notario-Pérez ${ }^{\mathrm{e}}$, Tamara del Corral ${ }^{\mathrm{a}, \mathrm{b}}$, \\ Bernardo Ramos-Díaz ${ }^{\mathrm{a}}$, Mario Acuyo-Osorio ${ }^{\mathrm{a}}$ and Roy La Touche ${ }^{\mathrm{a}, \mathrm{b}, \mathrm{c}, \mathrm{d}}$ \\ ${ }^{a}$ Departamento de Fisioterapia. Centro Superior de Estudios Universitarios La Salle, \\ Universidad Autónoma de Madrid, Madrid, Spain \\ ${ }^{\mathrm{b}}$ Motion in Brains Research Group, Centro Superior de Estudios Universitarios La Salle, \\ Universidad Autónoma de Madrid, Madrid, Spain \\ ${ }^{\mathrm{c}}$ Instituto de Neurociencia y dolor craneofacial (INDCRAN), Madrid, Spain \\ ${ }^{\mathrm{d}}$ Hospital La Paz Institute for Health Research (IdiPAZ), Madrid, Spain \\ ${ }^{\mathrm{d}}$ Regimiento de Artillería Antiaérea $n^{\circ} 71$ Acuartelamiento de Artillería de Fuencarral \\ "Capitán Guiloche”, Madrid, Spain
}

Received 22 June 2016

Accepted 23 March 2017

\begin{abstract}
.
BACKGROUND: Neck pain is one of the largest health problems in the military sector.

OBJECTIVE: To assess differences in the strength and endurance of the cervical muscles between military personnel with chronic nonspecific neck pain (CNSNP) with higher level of kinesiophobia (CNSNP-K) and individuals with lower levels of kinesiophobia (CNSNP-NK).

METHODS: We used kinesiophobia as a classification method: (1) CNSNP-K and (2) CNSNP-NK. The variables measured were endurance and strength of cervical muscles; range of motion (ROM), disability, pain intensity and psychological factors. RESULTS: Eighty-three military personnel (26 CNSNP-K; 20 CNSNP-NK and 37 asymptomatic). Statistically significant differences in endurance and ROM were only found between the CNSNP-K group and the control group. In strength and disability differences were revealed between both symptomatic groups and the control group [CNSNP-K vs. control (flex-ext $p<0.001$ ); CNSNP-NK vs. control (flex $p=0.003$ ) and (ext $p<0.001)$ ]. For psychological variables, the CNSNP-K group showed differences compared with the CNSNP-NK (pain catastrophizing, $p=0.007$; anxiety and depression, $p<0.001$ ) and with the asymptomatic group (pain catastrophizing, $p=0.008$; anxiety and depression, $p<0.001$ ).

CONCLUSIONS: Military personnel with CNSNP-K have functional limitations and associated psychosocial factors compared with asymptomatic subjects, and showed greater associated psychological factors than CNSNP-NK group. Military personnel with CNSNP-NK only showed decreased strength with respect to those who were asymptomatic.
\end{abstract}

Keywords: Physical endurance, muscle strength, range of motion and psychosocial factors

*Address for correspondence: Prof. Ibai López-de-UraldeVillanueva, Departamento de Fisioterapia, Centro Superior de Estudios Universitarios La Salle (UAM), La Salle Campus
Madrid, Calle la Salle, 10, 2836 Madrid, Spain. Tel.: +34 917401 980; E-mail: ibai.uralde@gmail.com. 


\section{Introduction}

Back pain significantly affects quality of life, and it is associated with low levels of labor productivity and sick leave [1]. Despite the increase in medical expenses for studies of neck pain, proportional savings have not accrued in clinical outcomes $[2,3]$. Neck pain is one of the largest health problems in the military sector because of its prevalence $[4,5]$, and it can interfere with security/compliance of military work [6-8]. Even though neck pain etiology is unknown in the military population, there are factors that may contribute to perpetuating this pain: prolonged forced neck postures, vibration from vehicles (airplanes, helicopters, etc.), and equipment carried on one's head $[9,10]$. The majority of investigations in this area have studied neck pain in military personnel in the air force; this fact is possibly due to the stringent demands on neck muscles experienced during flight [11-13]. However, we suggest that studies of military personnel stationed on land are also necessary due to the paucity of such studies.

Research conducted on the military population suggests that functional limitations (muscle weakness, muscle fatigue, and/or decreased range of motion (ROM)) in the cervical region could execute a crucial role in the development/maintenance of neck pain [14-16]. There is evidence showing that military personnel with neck pain have increased muscle fatigue and a reduced ROM in the cervical region [14, 17-20]. There are contradictory outcomes with respect to a decreased maximum voluntary contraction (MVC) of cervical muscles in military personnel with neck pain versus individuals without pain [14, $18,19,21,22]$. Studies in the literature postulate an equal force of the cervical muscles between symptomatic and asymptomatic military personnel [18, $19,22]$. The lack of evidence of differences can be explained by the fact that chronic neck pain is multifactorial [23] and includes psychological factors in addition to the largely physical and external factors evaluated in the aforementioned studies. These psychological factors include pain-related fear, which can be assessed as fear of movement due to pain (i.e., kinesiophobia) [24]. The term kinesiophobia was introduced by Miller et al. and makes reference to "an excessive, irrational, and debilitating fear of physical movement and activity resulting from a feeling of vulnerability to painful injury or reinjury" $[25,26]$. According to a qualitative review [27], pain-related fear is associated with decreased physical performance, greater pain expectations and a personal perception of higher disability. In light of this situation, we hypothesize that military personnel with chronic nonspecific neck pain with higher level of kinesiophobia (CNSNP-K) present greater functional limitations than individuals with lower levels of kinesiophobia (CNSNP-NK) or individuals who are asymptomatic.

The aim of this study was to assess differences in the strength and endurance of the cervical muscles between military personnel with CNSNP-K and military personnel with CNSNP-NK; we also investigated the differences in these variables between the two symptomatic groups with an asymptomatic group. In addition, our secondary aim was to determine differences between these same groups in terms of ROM, disability, pain intensity and psychological variables (pain catastrophizing, anxiety and depression). Catastrophizing is one cognitive factor that involves an exaggerated negative appraisal toward pain stimuli and pain experience [28]; furthermore, this cognitive factor is affected by anxiety and negative affectivity [28, 29].

\section{Methods}

\subsection{Study design}

We conducted an observational, cross-sectional, descriptive study. The study was developed in the flak barrack of Fuencarral "Capitán Guiloche" (Crta. de Fuencarral-Alcobendas, Madrid) during September and October 2015. In addition, the study was approved by the Ethics Committee of the Centro Superior de Estudios Universitarios La Salle and by the medical Center of the flak barrack of Fuencarral "Capitán Guiloche".

The research team was composed of two physiotherapists and one medical professional. The physician was responsible for assessing whether the participants satisfied the inclusion criteria. The physiotherapists performed the fieldwork, so they were responsible for assessing the different variables of the study. Both of the physiotherapists were trained in the protocol management for three hours. The training was aimed at performing an appropriate measurement of the different variables included in this study. This protocol was intended to minimize the potential for measurement bias.

\subsection{Study population}

The participants were recruited from flak barrack of Fuencarral "Capitán Guiloche" and they were 
military personnel belonging to ground force. The study population consisted of military personnel with chronic nonspecific neck pain and asymptomatic military personnel. The symptomatic military personnel included in the study were men and women with ages between 18 and 65 years with pain persisting for more than three months in the back of the neck (located between the occipital area and shoulders). Three months is considered a chronic pain according to some authors [30,31]. In addition, the symptomatic military personnel had to experience chronic neck pain of unknown etiology — nonspecific chronic neck pain - with symptoms provoked by prolonged neck postures/movements, or palpation of the neck musculature. Moreover, we required that participants not present any kind of cognitive impairment and that they possess an adequate ability to read, understand and speak Spanish.

Military personnel with chronic nonspecific neck pain were excluded from the study if they satisfied any of the following criteria: the presence of systemic diseases such as rheumatoid arthritis or cancer; a diagnosis of fibromyalgia; a history of whiplash, cervical radiculopathy or myelopathy; a history of cervical spine surgery; or the presence of dizziness/vertigo. Personnel were also excluded if they had received pain treatment in the cervical region, either physical therapy or medical treatment, within the last three months.

The asymptomatic control group was composed of military personnel aged 18-65 years who did not have a history of pain in the orofacial, cervical and/or thoracic region, and/or pain in their upper extremities within the last 12 months.

\subsection{Dependent variable}

\subsubsection{Kinesiophobia}

Fear of movement or re-injury due to pain was measured using the validated Spanish version of the Tampa Scale of Kinesiophobia (TSK-11). This scale consisting of 11 items, and each item is associated with a 4-point Likert scale ( 1 = "strongly disagree", $4=$ "strongly agree"). Higher scores correspond with a greater fear of pain, movement and injury. The Spanish version of the TSK-11 presents acceptable psychometric properties [32].

In addition, we used kinesiophobia as a classification method to divide the symptomatic participants into two groups: (1) military personnel with CNSNP$\mathrm{K}$ and (2) military personnel with CNSNP-NK. To carry out this division process, we used the cut-off scores based on the median scores obtained in the TSK-11 for all of the symptomatic military personnel. The individuals included in the CNSNP-K group exhibited a TSK-11 score at least equal to the median score, and the remaining individuals were placed into the CNSNP-NK group. This classification method has been used in previous studies [33].

\subsection{Primary outcomes}

\subsubsection{Endurance of cervical muscles}

Deep neck flexor endurance test $[34,35]$ was used to assess the endurance of this musculature. The participants were placed in supine decubitus with their hips and knees flexed and the soles of their feet resting on a table and their hands on their abdomens. Then, the examiner conducted the next movement sequence in the following order: 1) cranio-cervical flexion; 2) raised the participant's head above $2.5 \mathrm{~cm}$ on above the table; 3 ) indicated to the participant that he/she should maintain this position for as long as possible; and 4) withdrew his/her hands contact, leaving the head sustained only by the participant's muscular activity. The test ended when the participant wavered or lost cranio-cervical flexion. Finally, the time that the participant was able to maintain the test position was recorded. Deep neck flexor endurance test presents excellent intra-examiner reliability [34-36].

\subsubsection{Strength of cervical muscles}

The MVC force of the flexor and extensor muscles was examined using an analog dynamometer (Baseline ${ }^{\circledR}$ Evaluation Instrument, Fabrication Enterprises Inc. NY, USA). Each participant first performed a warm-up protocol to prepare his/her neck and shoulder muscles [37]. The test was performed with the subject in a sitting position with his/her arms beside his/her body. The subject was supported against a backrest and fixed to the backrest using a strap placed at the xiphoid process [38]. In addition, the participant was placed in neutral craniocervical position when the contraction started. We attempted to standardize the measurement process and minimize any compensation with axial musculature outside the cervical region.

The MVC force measurement of the flexor muscles was performed first, followed by the MVC force of the extensor muscles. To measure the flexion movement, we placed the bending head dynamometer on the frontal bone of the participant; for the extension test, we placed the bending head dynamometer on 
the occipital bone. Next, the participant performed a maximum-intensity contraction for 5 seconds a total of three times separated by 60 seconds of rest $[39,40]$. If the difference in force among the three trials was greater than $10 \%$, we proceeded to take another measurement [39]. We used the highest value of MVC force in our analysis. The contraction was isometric since no shortening or elongation of the musculature was recorded. Dynamometry has been shown to be a reliable method for assessing the MVC force of the cervical muscles [41-43].

\subsection{Secondary outcomes}

\subsubsection{Cervical range of motion}

We used a cervical range of motion (CROM) device (Performance Attainment Associates, Lindstrom, MN, USA) [44] to evaluate CROM. This device consists of three independent inclinometers, one for each plane of motion, fixed to a plastic frame resembling eyeglasses. Each participant sat in a chair with a backrest with their feet flat on the floor and their arms hanging alongside their body. We measured the following physiological movements: 1) flexion, 2) extension, 3) right lateral flexion, 4) left lateral flexion, 5) right rotation and 6) left rotation. Every physiological movement was repeated three times with 30 seconds of rest. The CROM is valid and reliable for measuring $\mathrm{ROM}$ in the cervical region [45-48].

\subsubsection{Pain intensity}

We used the Visual Analogue Scale (VAS) to measure pain intensity. This scale consists of a $10-\mathrm{cm}$ horizontal line with pain descriptors marked "no pain" on the left side and "the worst pain imaginable" on the right side. The VAS has been shown to be a valid and reliable tool to measure pain intensity $[49,50]$.

\subsubsection{Neck disability}

The perceived neck disability was evaluated using the validated Spanish version of the Neck Disability Index (NDI) [51]. This self-completed questionnaire consists of 10 items with six possible answers to each item. Scores range from 0 (no disability) to 5 (complete disability) points. The total score is calculated by adding up the responses for each item; the total maximum score is 50 points. The Spanish version of the NDI represents a valid, reliable and sensible tool to measure pain and disability of the neck [51].

\subsubsection{Pain catastrophizing}

The degree of pain catastrophizing was assessed using the Spanish version of the Pain Catastrophizing Scale (PCS) [52]. This scale is composed of 13 items, which must be answered with a numerical value between 0 (not at all) and 4 (all the time). The maximum score of the PCS is 52 points, where higher scores correspond to more pain catastrophizing. The Spanish version of PCS has demonstrated acceptable psychometric properties [52].

\subsubsection{Anxiety and depression}

We measured anxiety and depression levels using the validated Spanish version of the Hospital Anxiety and Depression Scale (HADS) [53]. The HADS consists of 14 items divided into two subscales for anxiety and depression. Both subscales include 7 items each, and the range of scores is $0-42$. A higher score is correlated with higher anxiety/depression levels. The Spanish version of the HADS is a valid and reliable screening tool to assess the detection of anxiety disorders and depression [53].

\subsection{Statistical analysis}

Statistical analysis was performed using the Statistical Package for Social Sciences (SPSS 21, SPSS Inc., Chicago, IL, USA). We used the KolmogorovSmirnov test to analyze the normal distribution of the variables. The data for continuous variables are presented as means \pm standard deviation (SD), median (1st and 3rd quartiles), 95\% confidence intervals (CI), and categorical variables as absolute (number) and relative frequency [n (\%)]. We used a chi-squared test to compare categorical variables. For the continuous parametric data, we used one-way ANOVA to analyze the group factor. To estimate the ANOVA effect size, we calculated the partial eta-squared $\left(\eta^{2}\right.$ p), and we classified the magnitude of the effect as small (0.01-0.059), medium (0.06-0.139), or large (>0.14) [54]. We performed post-hoc analysis with Bonferroni corrections in the case of significant ANOVA findings. We calculated effect sizes according to Cohen's method (Cohen's $d$ ), in which the magnitude of the effect was classified as small (0.20-0.49), medium (0.50-0.79), or large $(\geq 0.8)[55]$.

For the continuous nonparametric data, we used the Kruskal-Wallis test to assess the group factor. In this case, we used the Mann-Whitney U test to evaluate differences between groups. We did not find any statistically significant differences in the side factor 
for the CROM. For this reason, we added together the scores obtained in the CROM for the right side and the left side. We accordingly created the following variables: (1) total rotation and (2) total lateral flexion. For all of the analyses, we adopted a significance level of $5 \%(p<0.05)$ as corresponding to statistical significance.

\section{Results}

A total of 99 individuals were interested in participating in the study, and we excluded 16 of these individuals for not satisfying the inclusion criteria ( 5 cervical hernia diagnoses, 4 histories of whiplash, 2 cases of dizziness and 5 cases of cervical pain lasting for fewer than three months). The final sample consisted of 83 military personnel — military rank: private and corporal- with a mean age of $34.55 \pm 7.89$ years. The cohort was predominantly male (70 men and 13 women). The military personnel with chronic nonspecific neck pain presented exhibited a median (1st and 3rd quartile) score of 26 (IQR 20 to 28 ) on the TSK-11. All in all, the sample was distributed among 26 military personnel with CNSNP-K (21 men and 5 women), 20 military personnel with CNSNP-NK (15 men and 5 women), and 37 asymptomatic military personnel (34 men and 3 women). All of the variables were normally distributed except for MVC (flexion and extension). We did not find any statistically significant differences between the general characteristics of the three groups. In addition, the level of physical activity of each group was similar because all military personnel performed one hour of exercise per day. The descriptive characteristics of the participants in each group are listed in Table 1.

\subsection{Endurance and strength of cervical muscles}

One-way ANOVA revealed statistically significant differences in deep neck flexor endurance $(F=6.188$; $\left.p=0.003 ; \eta^{2}=0.134\right)$. We only found statistically significant differences between CNSNP-K and the asymptomatic group, where the effect size was $d=-0.83$. We did not detect any statistically significant differences between the symptomatic groups or between the CNSNP-NK and asymptomatic controls. The mean $\pm \mathrm{SD}$ and the mean difference $(95 \% \mathrm{CI})$ of the three groups are summarized in Table 2.

With respect to the MVC, the Kruskal-Wallis test revealed statistically significant differences for the flexion and extension movements. We found statistically significant differences in both movements between the control group and the two symptomatic groups; however, we did not observe any differences between the two pain groups $(p>0.05)$. Table 3 presents the median and 1st and 3rd quartiles, as well as the means $\pm \mathrm{SD}$ of each group.

\subsection{Range of motion, disability, pain intensity and psychological factors}

We found statistically significant differences in the group factor for all secondary outcomes [flexion $\left(\mathrm{F}=4.430 ; p=0.015 ; \eta^{2} \mathrm{p}=0.100\right)$; total lateral flexion $\left(\mathrm{F}=3.349 ; p=0.04 ; \eta^{2} \mathrm{p}=0.077\right)$; total rotation $\left(\mathrm{F}=4.024 ; p=0.022 ; \eta^{2} \mathrm{p}=0.091\right) ; \mathrm{NDI}(\mathrm{F}=41.211$; $\left.p<0.001 ; \eta^{2}=0.507\right)$; VAS $(\mathrm{F}=53.435 ; p<0.001$; $\left.\eta_{\mathrm{p}}^{2}=0.549\right) ; \mathrm{PCS}\left(\mathrm{F}=6.459 ; p=0.003 ; \eta_{\mathrm{p}}^{2}=0.139\right)$; HADS $\left.\left(\mathrm{F}=14.340 ; p<0.001 ; \eta^{2}{ }_{\mathrm{p}}=0.264\right)\right]$ except for extension CROM [extension $(\mathrm{F}=1.732 ; p=$ $\left.\left.0.183 ; \eta^{2} \mathrm{p}=0.042\right)\right]$.

Post-hoc testing with Bonferroni corrections revealed statistically significant differences between military personnel with CNSNP-K and asymptomatic military personnel for the movements of flexion, lateral flexion and rotation. The effect sizes for these movements were medium, but the effect size for flexion was the largest $(d=-0.76)$. In terms of the psychological variables (PCS and HADS), we recovered statistically significant differences between the

Table 1

Demographic characteristics of participants. Values are mean $\pm \mathrm{SD}$ and $n(\%)$

\begin{tabular}{lcccc}
\hline Characteristics & $\begin{array}{c}\text { CNSNP-K } \\
(n=26)\end{array}$ & $\begin{array}{c}\text { CNSNP-NK } \\
(n=20)\end{array}$ & $\begin{array}{c}\text { CG } \\
(n=37)\end{array}$ & $P$ values \\
\hline Age (years) & $35.68 \pm 9.49$ & $32.74 \pm 4.01$ & $34.74 \pm 8.26$ & $0.47^{*}$ \\
Gender (male) & $21(80.8)$ & $15(75)$ & $34(91.9)$ & $0.21^{\dagger}$ \\
Height (cm) & $170.28 \pm 8.48$ & $173.47 \pm 10.01$ & $175.82 \pm 8.74$ & $0.071^{*}$ \\
Weight $(\mathrm{kg})$ & $74.32 \pm 14.45$ & $76.71 \pm 14.63$ & $79.82 \pm 12.16$ & $0.30^{*}$ \\
\hline
\end{tabular}

CNSNP-K, chronic nonspecific neck pain with kinesiophobia; CNSNP-NK, chronic nonspecific neck pain without kinesiophobia; CG, control group. *Independent-samples Analysis of variance (ANOVA). ${ }^{\dagger} \chi^{2}$ tests. 
Table 2

Descriptive data and multiple comparisons for deep neck flexor endurance test and secondary outcomes

\begin{tabular}{|c|c|c|c|c|}
\hline \multirow[t]{2}{*}{ Outcomes } & \multicolumn{3}{|c|}{ Mean \pm SD } & \multirow{2}{*}{$\begin{array}{l}\text { Mean difference (95\% CI); Effect size }(d) \\
\text { a) CNSNP-K vs. CNSNP-NK } \\
\text { b) CNSNP-K vs. CG } \\
\text { c) CNSNP-NK vs. CG }\end{array}$} \\
\hline & CNSNP-K & CNSNP-NK & $\mathrm{CG}$ & \\
\hline DNFET $(\mathrm{sec})$ & $22.08 \pm 8.65$ & $25.6 \pm 9.67$ & $28.7 \pm 5.03$ & $\begin{array}{l}\text { a) }-3.52(-8.88 \text { to } 1.84) ; d=-0.31 \\
\text { b) }-6.62(-11.24 \text { to }-2.01)^{* *} ; d=-0.83 \\
\text { c) }-3.1(-8.1 \text { to } 1.9) ; d=-0.40\end{array}$ \\
\hline ROM-flexion $\left({ }^{\circ}\right)$ & $39.31 \pm 10.46$ & $39.73 \pm 15.05$ & $47.01 \pm 9.78$ & $\begin{array}{l}\text { a) }-0.42(-8.74 \text { to } 7.9): d=-0.03 \\
\text { b) }-7.7(-14.86 \text { to }-0.54)^{*}: d=-0.76 \\
\text { c) }-7.28(-15.05 \text { to } 0.49): d=-0.57\end{array}$ \\
\hline ROM-total lateral flexion $\left({ }^{\circ}\right)$ & $74.2 \pm 14.35$ & $76.66 \pm 17.03$ & $84.04 \pm 15.84$ & $\begin{array}{l}\text { a) }-2.47(-13.88 \text { to } 8.94): d=-0.16 \\
\text { b) }-9.85 \text { ( }-19.66 \text { to }-0.03)^{*}: d=-0.65 \\
\text { c) }-7.38(-18.03 \text { to } 3.26): d=-0.45\end{array}$ \\
\hline ROM-total rotation $\left({ }^{\circ}\right)$ & $122.42 \pm 18.22$ & $126.77 \pm 22.75$ & $134.77 \pm 13.24$ & $\begin{array}{l}\text { a) }-4.36(-17.07 \text { to } 8.36): d=-0.21 \\
\text { b) }-12.36(-23.3 \text { to }-1.42)^{*}: d=-0.75 \\
\text { c) }-8(-19.9 \text { to } 3.87): d=-0.43\end{array}$ \\
\hline NDI $(0-50)$ & $8.15 \pm 3.4$ & $7.55 \pm 4.26$ & $1.73 \pm 1.87$ & $\begin{array}{l}\text { a) } 0.6(-1.64 \text { to } 2.85): d=0.16 \\
\text { b) } 6.42(4.5 \text { to } 8.35)^{* *}: d=2.34 \\
\text { c) } 5.82(3.73 \text { to } 7.91)^{* *}: d=1.77\end{array}$ \\
\hline VAS $(0-10 \mathrm{~cm})$ & $2.76 \pm 1.91$ & $2.61 \pm 1.28$ & - & $\begin{array}{l}\text { a) }-0.15 \text { ( }-0.75 \text { to } 1.05) ; d=0.09 \\
\text { b) }- \\
\text { c) }-\end{array}$ \\
\hline PCS $(0-52)$ & $10.77 \pm 6.82$ & $5.4 \pm 5.29$ & $6.24 \pm 5.1$ & $\begin{array}{l}\text { a) } 5.37(1.2 \text { to } 9.54)^{* *}: d=0.88 \\
\text { b) } 4.53(0.94 \text { to } 8.11)^{* *}: d=0.75 \\
\text { c) }-0.84(-3.05 \text { to } 4.73): d=-0.16\end{array}$ \\
\hline HADS $(0-42)$ & $8.96 \pm 3.58$ & $4.85 \pm 2.83$ & $5.16 \pm 2.84$ & $\begin{array}{l}\text { a) } 4.11(1.86 \text { to } 6.36)^{* *}: d=1.27 \\
\text { b) } 3.8(1.86 \text { to } 5.73)^{* *}: d=1.18 \\
\text { c) }-0.31(-2.41 \text { to } 1.79): d=-0.11\end{array}$ \\
\hline
\end{tabular}

CNSNP-K, chronic nonspecific neck pain with kinesiophobia; CNSNP-NK, chronic nonspecific neck pain without kinesiophobia; CG, control group; DNFET, deep neck flexor endurance test; ROM, range of motion; NDI, neck disability index; VAS, visual analogue scale; PCS, pain catastrophizing scale; HADS, hospital anxiety and depression scale. ${ }^{*} p<0.05 .{ }^{* *} p<0.01$.

Table 3

Descriptive data and multiple comparisons for maximum voluntary contraction of the neck muscles

\begin{tabular}{|c|c|c|c|c|c|}
\hline $\mathrm{MVC}$ & CNSNP-K & CNSNP-NK & $\mathrm{CG}$ & Kruskal-Wallis & $\begin{array}{l}\text { U- Mann-Whitney } \\
\text { a) CNSNP-K vs. CNSNP-NK } \\
\text { b) CNSNP-K vs. CG } \\
\text { c) CNSNP-NK vs. CG }\end{array}$ \\
\hline \multirow[t]{4}{*}{ Flexion (kg) } & \multicolumn{3}{|c|}{ Median (1st and 3rd quartile) } & & \\
\hline & $4(1$ and 8$)$ & $4(2$ and 8$)$ & $8(6$ and 10$)$ & $<0.001$ & $\begin{array}{l}\text { a) } 0.383 \\
\text { b) }<0.001^{* *} \\
\text { c) } 0.003^{* *}\end{array}$ \\
\hline & & Mean \pm SD & & & \\
\hline & $4.38 \pm 3.31$ & $5.11 \pm 3.3$ & $7.76 \pm 2.39$ & - & - \\
\hline \multirow[t]{4}{*}{ Extension $(\mathrm{kg})$} & \multicolumn{3}{|c|}{ Median (1st and 3rd quartile) } & & \\
\hline & $6(2$ and 8$)$ & $6(4$ and 8$)$ & $10(8$ and 12$)$ & $<0.001$ & $\begin{array}{l}\text { a) } 0.496 \\
\text { b) }<0.001^{* *} \\
\text { c) }<0.001^{* *}\end{array}$ \\
\hline & \multicolumn{3}{|c|}{ Mean \pm SD } & & \\
\hline & $5.71 \pm 3.5$ & $6.32 \pm 2.99$ & $9.65 \pm 2.74$ & - & - \\
\hline
\end{tabular}

CNSNP-K, chronic nonspecific neck pain with kinesiophobia; CNSNP-NK, chronic nonspecific neck pain without kinesiophobia; CG, control group; MVC, maximal voluntary contraction. ${ }^{* *} p<0.01$.

CNSNP-K group and the CNSNP-NK group, as well as between CNSNP-K group and the asymptomatic group; we found the largest effect sizes in HADS (1.27 and 1.18, respectively). In addition, we found statistically significant differences in both chronic nonspecific neck pain groups compared with the asymptomatic group for the NDI variable with large effect sizes $(d>1.76)$. The mean \pm SD and mean differences $(95 \% \mathrm{CI})$ of the secondary outcomes of each group are listed in Table 2 . 


\section{Discussion}

Studies evaluating neck pain and its possible consequences on motor function in military populations have focused almost exclusively on air force personnel [14-22] as opposed to other military personnel such as individuals serving in land-based armies. In addition, most of these studies have taken into account only differences between military personnel with pain and asymptomatic individuals (i.e., psychological aspects have not been considered). The psychological aspects are crucial due to the multifactorial nature of pain, especially chronic pain. To the best of our knowledge, this study is the first to evaluate differences between army soldiers with and without chronic nonspecific neck pain as a function of their level of kinesiophobia. Thus, we believe that this manuscript is of general scientific interest.

\subsection{Endurance and strength of cervical muscles}

Our findings reveal that the role of kinesiophobia is relevant in terms of endurance/fatigue but not for cervical musculature force. In fact, military personnel with CNSNP-K only exhibited less endurance than individuals without pain. This difference could be considered clinically relevant since it is associated with a large effect size $(d=-0.83)$ [56, 57]. In contrast with previous investigations that demonstrated reduced endurance of cervical muscles in patients with reduced levels of pain [58-61], we found that military personnel with CNSNP-NK had the same endurance as individuals in the asymptomatic group. Our findings question a vision based exclusively on structural aspects [62, 63], reinforcing the theory that maintaining a contraction over time is dependent on both physiological and psychological factors [64-66]. The presence of more kinesiophobia was not determinative of the strength of the cervical musculature. In fact, our results reveal that military personnel with chronic nonspecific neck pain have less strength in their neck muscles, which is consistent with numerous lines of evidence developed in the civilian population [67-70]. Nevertheless, the literature about the military population is highly controversial $[14,18,19,21,22]$, and is in favor of the absence of differences in the strength of cervical muscles between military personnel with and without pain $[18,19,22]$. The lack of agreement between these studies and our findings may be explained because none of the studies took into account psychological factors such as kinesiophobia. In addition, these studies were conducted on air force military personnel, and the physical demands of these individuals may have differed from those of land-based forces [71].

Therefore, based on our results, kinesiophobia affects endurance but not the strength of the neck muscles. This finding may be explained by the fact that fear of movement does not have a large influence on MVC since it is a momentarily isometric contraction. Some researchers have confirmed this lack of an association between kinesiophobia and muscle strength in patients with back pain $[25,72,73]$. However, the MVC values that we obtained were the smallest in the CNSNP-K group, although the differences were not statistically significant. On the other hand, holding a position to assess the resistance of the neck muscles could be altered for the following reasons: 1) fear to injury of the patient, and 2) lower activity in their daily lives and therefore a less-optimum physical condition. Despite our assumptions, current scientific evidence has not found an association between kinesiophobia and the level of physical activity in patients with chronic nonspecific neck pain $[74,75]$. A recent research by Demirbüken et al. found that this association was presented in women with non-specific chronic neck pain but not in men [75]. It would be interesting for future studies carried out in military population to analyze the influence of gender on physical and psychological variables.

\subsection{Range of motion, disability, pain intensity and psychological factors}

This research has revealed that kinesiophobia also affects ROM because military personnel with CNSNP-K had a reduced ROM score compared to asymptomatic individuals. There is a vast body of literature demonstrating a reduced ROM in patients with pain $[67,76]$, so it is surprising that we did not note any differences between the CNSNP-NK group and the control group. It is noteworthy that, logically, military personnel with pain ought to exhibit more disability than asymptomatic individuals, but the disability was slight [77]. This value is smaller than the one reported in other neck pain studies in civilian populations. The known link between disability and functional limitation $[59,76,78]$, may explain the absence of differences. However, we found a reduced ROM in the CNSNP-K group despite these individuals having a mild disability. This finding may stem from military personnel with the highest level of 
kinesiophobia not reaching their full ROM in light of fear of injury (rather than a purely physical reason). In addition, the kinesiophobia could have a greater influence over the restriction of ROM in other types of neck pain such as the related to whiplash, since other investigations have shown that fear of movement has a significant impact in patients who have suffered a whiplash $[79,80]$.

Finally, military personnel with CNSNP-K exhibited stronger psychological effects compared than asymptomatic controls and individuals with CNSNPNK. In light of the demonstrated relationship between kinesiophobia and pain catastrophizing, anxiety and depression, this outcome was predictable [32,81-83].

\subsection{Clinical implications}

The effectiveness of the neck muscle training programs to relieve neck pain in the military population has been demonstrated [18, 84]. Nevertheless, the optimal way to perform such physical conditioning is not fully known, and researchers have argued that training focused on endurance or strength may have more benefits within a military population. The findings of this study allow us to hypothesize that military personnel with the highest level of kinesiophobia may possibly obtain more benefits from endurance training; this question can be answered quickly using the TSK-11. Moreover, a training protocol with a cognitive-behavioral approach (e.g., the inclusion of coping strategies) may produce more benefits among military personnel with CNSNP$\mathrm{K}$ in order to reduce their kinesiophobia. In fact, a recent study has shown that a physical training program combined with a cognitive-behavioral treatment can reduce work-related fear-avoidance beliefs in patients with musculoskeletal pain including neck pain [85]. Future studies that include therapeutic education based on a cognitive-behavioral approach with a cervical muscle training program are necessary.

\subsection{Limitations}

The primary limitation was the cross-sectional design of this research, which prevents us from clearly establishing the cause of the results. Another limitation was that cervical muscular endurance was not assessed using a more sophisticated method (e.g., surface electromyography). Even so, the reliability of the electromyography in the cervical region is controversial because of the large number of existing muscles [86]. Furthermore, we opted to use the deep neck flexors endurance test since it was an easier method to apply in clinical practice. In addition, this study was conducted without a sample size calculation because the initial objective was to evaluate the largest possible proportion of military personnel from the Acuartelamiento de Artillería de Fuencarral "Capitán Guiloche"; however, this situation may be considered to be a limitation. Finally, due to the size of the sample and the small number of women participating in the study, it was not possible to perform a statistical analysis taking into account the gender factor. Additional studies taking into account the limitations found in this investigation are necessary.

\section{Conclusions}

The results of this study demonstrate that military personnel with CNSNP-K have functional limitations and associated psychosocial factors compared with asymptomatic military personnel. Nonetheless, military personnel with CNSNP-NK only demonstrated a strength reduction in cervical muscles compared with individuals without pain. There were only differences between the two groups with pain in terms of psychological variables. Additional studies are necessary to confirm these results.

\section{Acknowledgments}

The authors like to thank all members of the flak barrack of Fuencarral "Capitán Guiloche" who made this study possible, specially to Colonel José Luis García Madrid and Commandant José Pablo Rodríguez de la Paz Navarro. The authors also wish to thank Spanish armed forces for their support as well as for their willingness to contribute to the development and scientific advance. The views, opinions, findings, conclusions and recommendations contained in this study are those of the authors and are not necessarily endorsed by the Spanish armed force.

The study protocol was approved by the Human Subjects Review Committee of the Centro Superior de Estudios Universitarios La Salle in Madrid, Spain.

\section{Conflict of interest}

The authors certify that they have no affiliations with or financial involvement in any organization or 
entity with a direct financial interest in the subject matter or materials discussed in the article.

\section{References}

[1] Dagenais S, Caro J, Haldeman S. A systematic review of low back pain cost of illness studies in the United States and internationally. Spine J 2008;8(1):8-20.

[2] Driessen M, Bosmans J, Proper K, Anema J, Bongers P, van der Beek A. The economic evaluation of a participatory ergonomics programme to prevent low back and neck pain. Work 2012;41(Suppl 1):2315-20.

[3] Martin BI, Deyo RA, Mirza SK, Turner JA, Comstock BA, Hollingworth W, Sullivan SD. Expenditures and health status among adults with back and neck problems. JAMA 2008;299(6):656-64.

[4] Ang B, Harms-Ringdahl K. Neck pain and related disability in helicopter pilots: A survey of prevalence and risk factors. Aviat Space Environ Med 2006;77(7):713-9.

[5] Walters P, Cox J, Clayborne K, Hathaway A. Prevalence of neck and back pain amongst aircrew at extremes of anthropometric measurements. Ft Rucker US Army Aeromed Res Lab 2012;USAARL Rep.

[6] Lincoln AE, Smith GS, Amoroso PJ, Bell NS. The natural history and risk factors of musculoskeletal conditions resulting in disability among US Army personnel. Work 2002;18(2):99-113.

[7] Newman DG. +GZ-induced neck injuries in Royal Australian Air Force fighter pilots. Aviat Space Environ Med 1997;68(6):520-4.

[8] Thomae MK, Porteous JE, Brock JR, Allen GD, Heller RF. Back pain in Australian military helicopter pilots: A preliminary study. Aviat Space Environ Med 1998;69(5):468-73.

[9] Green ND. Acute soft tissue neck injury from unexpected acceleration. Aviat Space Environ Med 2003;74(10): 1085-90.

[10] Hämäläinen $\mathrm{O}$, Vanharanta H. Effect of $\mathrm{Gz}$ forces and head movements on cervical erector spinae muscle strain. Aviat Space Environ Med 1992;63(8):709-16.

[11] Burnett AF, Naumann FL, Burton EJ. Flight-training effect on the cervical muscle isometric strength of trainee pilots. Aviat Space Environ Med 2004;75(7):611-5.

[12] Hämäläinen O, Vanharanta H, Bloigu R. Determinants of +Gz-related neck pain: A preliminary survey. Aviat Space Environ Med 1993;64(7):651-2.

[13] Knudson R, McMillan D, Doucette D, Seidel M. A comparative study of G-induced neck injury in pilots of the F/A-18, A-7, and A-4. Aviat Space Environ Med 1988;59(8):758-60.

[14] Ang B, Linder J, Harms-Ringdahl K. Neck strength and myoelectric fatigue in fighter and helicopter pilots with a history of neck pain. Aviat Space Environ Med 2005;76(4): 375-80.

[15] Coakwell MR, Bloswick DS, Moser R. High-risk head and neck movements at high $G$ and interventions to reduce associated neck injury. Aviat Space Environ Med 2004; 75(1):68-80.

[16] De Loose V, Van den Oord M, Burnotte F, Van Tiggelen D, Stevens V, Cagnie B, Witvrouw E, Danneels L. Individual, work-, and flight-related issues in F-16 pilots reporting neck pain. Aviat Space Environ Med 2008;79(8):779-83.

[17] Ang BO. Impaired neck motor function and pronounced pain-related fear in helicopter pilots with neck pain - a clinical approach. J Electromyogr Kinesiol 2008;18(4):538-49.
[18] De Loose V, Van den Oord M, Burnotte F, Van Tiggelen D, Stevens V, Cagnie B, Danneels L, Witvrouw E. Functional assessment of the cervical spine in F-16 pilots with and without neck pain. Aviat Space Environ Med 2009;80(5):477-81.

[19] Nagai T, Abt JP, Sell TC, Clark NC, Smalley BW, Wirt MD, Lephart SM. Neck proprioception, strength, flexibility, and posture in pilots with and without neck pain history. Aviat Space Environ Med 2014;85(5):529-35.

[20] van den Oord MHAH, De Loose V, Meeuwsen T, Sluiter JK, Frings-Dresen MHW. Neck pain in military helicopter pilots: Prevalence and associated factors. Mil Med 2010;175(1):55-60.

[21] Lecompte J, Maisetti O, Guillaume A, Skalli W, Portero P. Neck strength and EMG activity in fighter pilots with episodic neck pain. Aviat Space Environ Med 2008;79(10): 947-52.

[22] Van den Oord MHAH, De Loose V, Sluiter JK, FringsDresen MHW. Neck strength, position sense, and motion in military helicopter crew with and without neck pain. Aviat Space Environ Med 2010;81(1):46-51.

[23] Bogduk N. The anatomy and pathophysiology of neck pain. Phys Med Rehabil Clin N Am 2003;14(3):455-72, v.

[24] Zale EL, Lange KL, Fields SA, Ditre JW. The relation between pain-related fear and disability: A meta-analysis. J Pain 2013;14(10):1019-30.

[25] Hudes K. The Tampa Scale of Kinesiophobia and neck pain, disability and range of motion: A narrative review of the literature. J Can Chiropr Assoc 2011;55(3):222-32.

[26] Miller R, Kori S, Todd D. The Tampa Scale: A measure of kinesiophobia. Clin J Pain 1991;7(1):51-2.

[27] Vlaeyen JW, Linton SJ. Fear-avoidance and its consequences in chronic musculoskeletal pain: A state of the art. Pain 2000;85(3):317-32.

[28] Turner JA, Aaron LA. Pain-related catastrophizing: What is it? Clin J Pain 2001;17(1):65-71.

[29] Park SJ, Lee R, Yoon DM, Yoon KB, Kim K, Kim SH. Factors associated with increased risk for pain catastrophizing in patients with chronic neck pain. Medicine (Baltimore) 2016;95(37):e4698.

[30] Binder AI. Neck pain. Clin Evid (Online) 2008;pii:1103.

[31] Jayson MI. Why does acute back pain become chronic? BMJ 1997;314(7095):1639-40.

[32] Gómez-Pérez L, López-Martínez AE, Ruiz-Párraga GT. Psychometric Properties of the Spanish Version of the Tampa Scale for Kinesiophobia (TSK). J Pain 2011;12(4): 425-35.

[33] Hudes K. The Tampa Scale of Kinesiophobia and neck pain, disability and range of motion: A narrative review of the literature. J Can Chiropr Assoc 2011;55(3):222-32.

[34] Domenech M, Sizer P, Dedrick G, McGalliard M, Brismee J. The deep neck flexor endurance test: Normative data scores in healthy adults. PM R 2011;3(2):105-10.

[35] Harris KD, Heer DM, Roy TC, Santos DM, Whitman JM, Wainner RS. Reliability of a measurement of neck flexor muscle endurance. Phys Ther 2005;85(12):1349-55.

[36] Edmondston SJ, Wallumrød ME, Macléid F, Kvamme LS, Joebges S, Brabham GC. Reliability of isometric muscle endurance tests in subjects with postural neck pain. J Manipulative Physiol Ther 2008;31(5):348-54.

[37] Saez Saez de Villarreal E, González-Badillo JJ, Izquierdo M. Optimal warm-up stimuli of muscle activation to enhance short and long-term acute jumping performance. Eur J Appl Physiol 2007;100(4):393-401.

[38] Dezman ZDW, Ledet EH, Kerr HA. Neck strength imbalance correlates with increased head acceleration in soccer heading. Sports Health 2013;5(4):320-6. 
[39] Strimpakos N, Sakellari V, Gioftsos G, Oldham J. Intratester and intertester reliability of neck isometric dynamometry. Arch Phys Med Rehabil 2004;85(8):1309-16.

[40] Levoska S, Keinänen-Kiukanniemi S, Hämäläinen O, Jäms $\mathrm{T}$, Vanharanta $\mathrm{H}$. Reliability of a simple method of measuring isometric neck muscle force. Clin Biomech (Bristol, Avon) 1992;7(1):33-7.

[41] Cadogan A, Laslett M, Hing W, McNair P, Williams M. Reliability of a new hand-held dynamometer in measuring shoulder range of motion and strength. Man Ther 2011;16(1):97-101.

[42] Geary K, Green BS, Delahunt E. Intrarater reliability of neck strength measurement of rugby union players using a handheld dynamometer. J Manipulative Physiol Ther 2013;36(7):444-9.

[43] Shahidi B, Johnson CL, Curran-Everett D, Maluf KS. Reliability and group differences in quantitative cervicothoracic measures among individuals with and without chronic neck pain. BMC Musculoskelet Disord 2012;13:215.

[44] Audette I, Dumas J-P, Côté JN, De Serres SJ. Validity and between-day reliability of the cervical range of motion (CROM) device. J Orthop Sports Phys Ther 2010;40(5):318-23.

[45] Williams MA, McCarthy CJ, Chorti A, Cooke MW, Gates S. A systematic review of reliability and validity studies of methods for measuring active and passive cervical range of motion. J Manipulative Physiol Ther 2010;33(2): 138-55.

[46] Garrett TR, Youdas JW, Madson TJ. Reliability of measuring forward head posture in a clinical setting. J Orthop Sports Phys Ther 1993;17(3):155-60.

[47] Fletcher JP, Bandy WD. Intrarater reliability of CROM measurement of cervical spine active range of motion in persons with and without neck pain. J Orthop Sports Phys Ther 2008;38(10):640-5.

[48] Youdas JW, Carey JR, Garrett TR. Reliability of measurements of cervical spine range of motion-comparison of three methods. Phys Ther 1991;71(2):98-104-6.

[49] Jensen MP, Turner JA, Romano JM, Fisher LD. Comparative reliability and validity of chronic pain intensity measures. Pain 1999;83(2):157-62.

[50] Katz J, Melzack R. Measurement of pain. Surg Clin North Am 1999;79(2):231-52.

[51] Andrade Ortega JA, Delgado Martínez AD, Almécija Ruiz R. Validation of the Spanish version of the Neck Disability Index. Spine (Phila Pa 1976) 2010;35(4):E114-8

[52] García Campayo J, Rodero B, Alda M, Sobradiel N, Montero J, Moreno S. Validation of the Spanish version of the Pain Catastrophizing Scale in fibromyalgia. Med Clin (Barc) 2008;131(13):487-92.

[53] Herrero MJ, Blanch J, Peri JM, De Pablo J, Pintor L, Bulbena A. A validation study of the hospital anxiety and depression scale (HADS) in a Spanish population. Gen Hosp Psychiatry 2003;25(4):277-83.

[54] Cohen J. Eta-squared and partial eta-squared in fixed factor ANOVA designs. Educ Psychol Meas 1973;33:107-12.

[55] Cohen J. Statistical power analysis for the behavioral sciences. 2nd ed. Hillsdale: New Jersey: Lawrence Earlbaum Associates; 1988.

[56] Page P. Beyond statistical significance: Clinical interpretation of rehabilitation research literature. Int J Sports Phys Ther 2014;9(5):726-36.

[57] Jaeschke R, Singer J, Guyatt GH. Measurement of health status. Ascertaining the minimal clinically important difference. Control Clin Trials 1989;10(4):407-15.
[58] Falla D, Jull G, Edwards S, Koh K, Rainoldi A. Neuromuscular efficiency of the sternocleidomastoid and anterior scalene muscles in patients with chronic neck pain. Disabil Rehabil 2004;26(12):712-7.

[59] Lee H, Nicholson LL, Adams RD. Neck muscle endurance, self-report, and range of motion data from subjects with treated and untreated neck pain. J Manipulative Physiol Ther 2005;28(1):25-32.

[60] Peolsson A, Kjellman G. Neck muscle endurance in nonspecific patients with neck pain and in patients after anterior cervical decompression and fusion. J Manipulative Physiol Ther 2007;30(5):343-50.

[61] Jull G, Kristjansson E, Dall'Alba P. Impairment in the cervical flexors: A comparison of whiplash and insidious onset neck pain patients. Man Ther 2004;9(2):89-94.

[62] Mannion AF, Dumas GA, Stevenson JM, Cooper RG. The influence of muscle fiber size and type distribution on electromyographic measures of back muscle fatigability. Spine (Phila Pa 1976) 1998;23(5):576-84

[63] Uhlig Y, Weber BR, Grob D, Müntener M. Fiber composition and fiber transformations in neck muscles of patients with dysfunction of the cervical spine. J Orthop Res 1995;13(2):240-9.

[64] Gardiner PF. Neuromuscular aspects of physical activity. 6 Ed. Champaign, IL: Human Kinetics; 2001.

[65] De Luca CJ. Use of the surface EMG signal for performance evaluation of back muscles. Muscle Nerve 1993;16(2): 210-6.

[66] Enoka R. Neuromechanics of Human Movement. 5 Ed. Champaign, IL: Human Kinetics; 2002.

[67] Cagnie B, Cools A, De Loose V, Cambier D, Danneels L. Differences in isometric neck muscle strength between healthy controls and women with chronic neck pain: The use of a reliable measurement. Arch Phys Med Rehabil 2007;88(11):1441-5.

[68] Chiu TTW, Sing K Lo. Evaluation of cervical range of motion and isometric neck muscle strength: Reliability and validity. Clin Rehabil 2002;16(8):851-8.

[69] Prushansky T, Gepstein R, Gordon C, Dvir Z. Cervical muscles weakness in chronic whiplash patients. Clin Biomech (Bristol, Avon) 2005;20(8):794-8.

[70] Ylinen J, Salo P, Nykänen M, Kautiainen H, Häkkinen A. Decreased isometric neck strength in women with chronic neck pain and the repeatability of neck strength measurements. Arch Phys Med Rehabil 2004;85(8):1303-8.

[71] Alricsson M, Harms-Ringdahl K, Schüldt K, Ekholm J, Linder J. Mobility, muscular strength and endurance in the cervical spine in Swedish Air Force pilots. Aviat Space Environ Med 2001;72(4):336-42.

[72] Demoulin C, Huijnen IPJ, Somville P-R, Grosdent S, Salamun I, Crielaard J-M, Vanderthommen M, Volders S. Relationship between different measures of pain-related fear and physical capacity of the spine in patients with chronic low back pain. Spine J 2013;13(9):1039-47.

[73] Verbunt JA, Seelen HA, Vlaeyen JW, Bousema EJ, van der Heijden GJ, Heuts PH, Knottnerus JA. Pain-related factors contributing to muscle inhibition in patients with chronic low back pain: An experimental investigation based on superimposed electrical stimulation. Clin J Pain 2005;21(3):232-40.

[74] Cheung J, Kajaks T, Macdermid JC. The relationship between neck pain and physical activity. Open Orthop J 2013;7(1):521-9.

[75] Demirbüken I, Özgül B, Kuru Çolak T, Aydoğdu O, Sari Z, Yurdalan SU. Kinesiophobia in relation to physical 
activity in chronic neck pain. J Back Musculoskelet Rehabil 2016;29(1):41-7.

[76] Nordin M, Carragee EJ, Hogg-Johnson S, Weiner SS, Hurwitz EL, Peloso PM, Guzman J, van der Velde G, Carroll LJ, Holm LW, Côté P, Cassidy JD, Haldeman S. Assessment of neck pain and its associated disorders: Results of the Bone and Joint Decade 2000-2010 Task Force on Neck Pain and Its Associated Disorders. Spine (Phila Pa 1976) 2008;33(4 Suppl):S101-22.

[77] Vernon H. The Neck Disability Index: State-of-the-art, 1991-2008. J Manipulative Physiol Ther 2008;31(7):491502.

[78] Hermann KM, Reese CS. Relationships among selected measures of impairment, functional limitation, and disability in patients with cervical spine disorders. Phys Ther 2001;81(3):903-14.

[79] Pedler A, Kamper SJ, Sterling M. Addition of posttraumatic stress and sensory hypersensitivity more accurately estimates disability and pain than fear avoidance measures alone after whiplash injury. Pain 2016;157(8):1645-54.

[80] Carriere JS, Thibault P, Milioto M, Sullivan MJL. Expectancies mediate the relations among pain catastrophizing, fear of movement, and return to work outcomes after whiplash injury. J Pain 2015;16(12):1280-7.

[81] Doménech J, Sanchis-Alfonso V, Espejo B. Changes in catastrophizing and kinesiophobia are predictive of changes in disability and pain after treatment in patients with anterior knee pain. Knee Surg Sports Traumatol Arthrosc 2014;22(10):2295-300.

[82] Domenech J, Sanchis-Alfonso V, López L, Espejo B. Influence of kinesiophobia and catastrophizing on pain and disability in anterior knee pain patients. Knee Surg Sports Traumatol Arthrosc 2013;21(7):1562-8.

[83] López-de-Uralde-Villanueva I, Beltran-Alacreu H, Fernández-Carnero J, Gil-Martínez A, La Touche R. Differences in Neural Mechanosensitivity Between Patients with Chronic nonspecific Neck Pain With and Without Neuropathic Features. A Descriptive Cross-Sectional Study. Pain Med 2015; [Epub ahead of print].

[84] Salmon DM, Harrison MF, Neary JP. Neck pain in military helicopter aircrew and the role of exercise therapy. Aviat Space Environ Med 2011;82(10):978-87.

[85] Jay K, Brandt M, Jakobsen MD, Sundstrup E, Berthelsen KG, Schraefel M, Sjøgaard G, Andersen LL. Ten weeks of physical-cognitive-mindfulness training reduces fearavoidance beliefs about work-related activity: Randomized controlled trial. Medicine (Baltimore) 2016;95(34):e3945.

[86] Strimpakos N. The assessment of the cervical spine. Part 2: Strength and endurance/fatigue. J Bodyw Mov Ther 2011;15(4):417-30. 